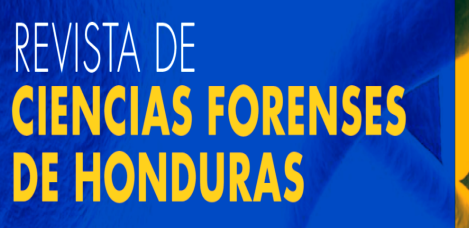

Revista de Ciencias Forenses de Honduras ISSN: 2412-8058

ISSN: 2413-1067

revistacienciasforenseshnd@gmail.com

Dirección de Medicina Forense de Honduras Honduras

\title{
Sabillón, Nicolas
}

A cuatro años de la fundación de la Revista de Ciencias Forenses de Honduras: Logros y retos Revista de Ciencias Forenses de Honduras, vol. 4, núm. 1, 2018, Enero-Junio, pp. 31-34

Dirección de Medicina Forense de Honduras Honduras 


\section{A cuatro años de la fundación de la Revista de Ciencias Forenses de Honduras: Logros y retos}

\section{Four years of the Journal of Forensics Sciences of Honduras : Achievements and challenges}

Nicolas Sabillón ${ }^{1}$

\footnotetext{
${ }^{1}$ Patólogo, Dirección de Medicina Forense, Jefe Departamento de Patología, UNAH.
}

Correspondencia a: nsabillon@gmail.com

\section{REFERENCIA:}

Sabillón N. A cuatro años de la fundación de la Revista de Ciencias Forenses de Honduras. Rev. cienc. forenses Honduras. 2018; 4(1): 31-34.

\section{RESUMEN}

La Revista de Ciencias Forense de Honduras fue fundada en el año 2015 a fin de llenar un vacío en las Ciencias Forenses Hondureñas, por lo que su creación representa un importante avance en el tema de divulgación de estas disciplinas, a pesar de su tímido inicio, la misma ha venido creciendo saludablemente, no sin enfrentar importantes desafíos que deben ser superados, si es que se espera la misma continúe creciendo y se convierta en un verdadero medio de difusión científica.

\section{PALABRAS CLAVE}

Revista de ciencias Forenses de Honduras, logros y retos, indexación, diseminación científica.

\section{ABSTRACT}

The Journal of Forensic Sciences of Honduras was founded in 2015 in order to fill a gap in Honduran forensic sciences, so its creation represents an important advance in the dissemination of these disciplines, despite its timid start, it has been growing healthily, not without facing important challenges that must be overcome, if it is expected to continue to grow and become a true means of scientific dissemination.

\section{KEYWORDS}

Journal of Forensic Sciences of Honduras, achievements and challenges, indexation. scientific dissemination.

\section{INTRODUCCIÓN}

En el presente año, 2018, la Revista de Ciencias Forenses de Honduras cumple cuatro años de publicación ininterrumpida. Esta revista nació bajo la visión de la actual Directora de Medicina Forense, la Dra Semma Julissa Villanueva, quien consideró que ya era tiempo que las Ciencias Forenses en Honduras contaran con un instrumento de difusión y divulgación científico, delegando la responsabilidad de su organización y dirección a la Dra. Mireya Matamoros Zelaya.

\section{LOGROS}

A los cuatro años de su inicio, es conveniente hacer una valoración de lo que se ha logrado y los retos que debe enfrentar. Entre los logros destacan: 
1. La conformación de un Consejo Editorial. Este Consejo lo integran personal de Medicina Forense de Honduras, de la Universidad Nacional Autónoma de Honduras y profesionales y médicos forenses de otros institutos. La actual Directora de la Revista es la Dra. Mireya Matamoros Zelaya.

2. La obtención de un código numérico reconocido internacionalmente para la identificación de la Revista. ISSN (International Standard Serial Number/Numero Internacional Normalizado de Publicaciones Seriadas. Este identifica la Revista en cualquier idioma en cualquier parte del mundo. La Revista tiene ISSN tanto para la versión impresa (2412 - 8058) como electrónica (2413 - 1067).

3. La Visibilidad en plataformas digitales. La Revista es visible en diferentes plataformas:

3a.-La plataforma de la Biblioteca Virtual en Salud (BVS Honduras, certificada por el Centro Latinoamericano y del Caribe de Información en Ciencias de la Salud, conocida por sus siglas como BIREME)

3b.-Indexada en Latindex (Directorio). Esta base de datos es el Sistema Regional de Información en Línea para Revistas Científicas de América Latina, el Caribe, España y Portugal. Es el índice más completo e importante de la literatura científica y técnica en salud de América Latina y del Caribe.

3c.-Actualmente se hacen gestiones para que sea visible en la Plataforma CAMJOL (Revistas en Línea Centroamericanas) y en el Catalogo de Latindex.
4. La Periodicidad. La Revista desde un inicio se ha publicado de forma semestral, dos números al año, con la publicación de los dos números del 2018, se encuentra actualizada.

5. La calidad de los artículos publicados. A fin de garantizar la calidad de lo que se publica, la Revista se apega y solicita a los autores redactar de acuerdo a los Requisitos Uniformes de los Manuscritos Propuestos para la Publicación en Revistas Biomédicas, conocidas como recomendaciones CMJE, se realiza revisión por pares. Se publican artículos científicos originales, casos clínicos, revisiones bibliográficas, artículos de opinión, entre otros. En los cuatro años, con el presente número, se han publicado 55 artículos, 13 de los cuales son originales, escritos por autores nacionales e internacionales.

Entre los artículos publicados, se pueden enumerar:

- Estudio médico - legal de muertes por electrocución (Instituto de Medicina Legal de Málaga, España).

- La dimensión sanitaria y social de la patología forense (Instituto de Medicina Legal de Sevilla, España).

- La profesionalización de los operadores del sector justicia y seguridad

- La prueba pericial en temas de contabilidad, auditoria y finanzas.

- La revictimización como causal de silencio de la víctima.

- La investigación pericial forense de los cuerpos mutilados (Instituto de Medicina Legal y Forense de Panamá). 
- El impacto de la antropología forense en Guatemala (Fundación de Antropología Forense de Guatemala).

- Factores humanos condicionantes claves para mejorar la seguridad vial en Honduras.

- Caracterización de casos de violencia infligida por la pareja evaluados en la Dirección de Medicina Forense de Tegucigalpa, entre 2010 y 2014.

- Una pareja explosiva: alcohol y conducción.

- Retos y oportunidades para el transporte regional de carga. La experiencia Crowley en Centroamérica

- Lo que se debe saber sobre la Histopatología Forense.

- Determinación de drogas de abuso en pelo.

- La violencia contra la mujer. ¿Un problema del área rural?

- Ciencias forenses y pruebas presuntivas 16. Conocimientos, actitudes y creencias sobre donación y trasplante en Honduras.

- 17. Importancia de los registros dentales antemortem en los centros penitenciarios.

- 18. Las pruebas de ADN en el contexto forense.

- 19.-Primer curso de redacción de artículos científicos en la Dirección de Medicina Forense de Honduras, entre otros.
6. La Participación del Postgrado de Medicina legal y Forense de Honduras: En el 2014, por gestiones de la Dra. Semma Julissa Villanueva, se creó el postgrado de medicina legal y forense en convenio con la UNAH. A la fecha han egresado cinco médicos especialistas de este postgrado y en el presente año egresaran cuatro; nueve especialistas en total desde su creación al 2018. Para la revista esto constituye un aporte fundamental, ya que espera nutrirse de los distintos trabajos científicos que realizan estos estudiantes.

7. La capacitación en redacción de artículos científicos. En vista del escaso/nulo conocimiento que existía en la Dirección de Medicina Forense de Honduras para redactar artículos científicos, el Consejo editorial con el apoyo de la Biblioteca Medica Nacional de la UNAH ha impartido y seguirá impartiendo cursos al personal de medicina forense para que redacten $y$ publiquen las investigaciones que realizan.

8. La colaboración de la Biblioteca Medica Nacional (BMN) de la UNAH. Este ha sido un pilar fundamental en el camino hasta hoy transitado. Esta colaboración se ha centrado en hacer visible la Revista a nivel nacional e internacional a través de plataformas digitales, tal prestigiosas como la BVS; así como apoyo en capacitación y asesoría en el área de edición.

\section{RETOS}

Si se consideran los retos, estos son muchos, se pueden resumir en lo siguiente: 
1.-SE DEBE MANTENER Y FORTALECER LO QUE HASTA AHORA SE HA LOGRADO. La Revista es institucional y lo ideal es que tenga mayor apoyo de parte del Ministerio Publico como institución. La sostenibilidad de una revista es una tarea difícil, depende de muchos factores (motivación del personal para que publique, reconocimiento de las publicaciones, recursos humanos, financieros, etc.), estos factores pueden ser superados con apoyo de la institución responsable.

2.- Debe fortalecerse la capacidad de generación de investigación y conocimiento en la Dirección de Medicina Forense, ya que al ser un ente eminentemente técnico la investigación debe conceptualizarse como un quehacer fundamental.

La Revista de Ciencias Forenses de Honduras ha venido a llenar un vacío desde hace mucho tiempo existente en el área de las ciencias forenses hondureñas. A cuatro años de su fundación ha tenido logros importantes. Los retos son muchos, con la motivación, capacitación del personal y apoyo institucional se puede superar lo realizado hasta hoy. 